function. Nat Immunol. 2011;12(9):888-897.

9. Wu Q, et al. The tuberous sclerosis complex-mammalian target of rapamycin pathway maintains the quiescence and survival of naive T cells. J Immunol. 2011;187(3):1106-1112.

10. O'Brien TF, et al. Regulation of T-cell survival and mitochondrial homeostasis by TSC1. Eur J Immunol. 2011;41(11):3361-3370.

11. Park Y, et al. TSC1 regulates the balance between effector and regulatory T cells. J Clin Invest. 2013; 123(12):5165-5178.

12. Shale M, Schiering C, Powrie F. CD4(+) T-cell subsets in intestinal inflammation. Immunol Rev. 2013;252(1):164-182.

13. Zhou X, et al. Instability of the transcription factor Foxp3 leads to the generation of pathogenic memory $\mathrm{T}$ cells in vivo. Nat Immunol. 2009;10(9):1000-1007.

14. Yu Y, et al. Phosphoproteomic analysis iden- tifies Grb10 as an mTORC1 substrate that negatively regulates insulin signaling. Science. 2011;332(6035):1322-1326.

15. Hsu PP, et al. The mTOR-regulated phosphoproteome reveals a mechanism of mTORC1-mediated inhibition of growth factor signaling. Science. 2011;332(6035):1317-1322.

16. Ouyang W, Beckett O, Ma Q, Paik JH, DePinho RA, Li MO. Foxo proteins cooperatively control the differentiation of Foxp3+ regulatory T cells. Nat Immunol. 2010;11(7):618-627.

17. Ouyang W, et al. Novel Foxo1-dependent transcriptional programs control $\mathrm{T}$ (reg) cell function. Nature. 2012;491(7425):554-559.

18. Kerdiles YM, et al. Foxo transcription factors control regulatory $\mathrm{T}$ cell development and function. Immunity. 2010;33(6):890-904.

19. Smith PM, et al. The microbial metabolites, short- chain fatty acids, regulate colonic Treg cell homeostasis. Science. 2013;341(6145):569-573.

20. Düvel K, et al. Activation of a metabolic gene regulatory network downstream of mTOR complex 1. Mol Cell. 2010;39(2):171-183.

21. Yang K, Chi H. mTOR and metabolic pathways in $\mathrm{T}$ cell quiescence and functional activation. Semin Immunol. 2012;24(6):421-428.

22. Zeng H, Chi H. mTOR and lymphocyte metabolism. Curr Opin Immunol. 2013;25(3):347-355.

23. Zeng H, Yang K, Cloer C, Neale G, Vogel P, Chi H. mTORC1 couples immune signals and metabolic programming to establish $\mathrm{T}(\mathrm{reg})$-cell function. Nature. 2013;499(7459):485-490.

24. Benjamin D, Colombi M, Moroni C, Hall MN. Rapamycin passes the torch: a new generation of mTOR inhibitors. Nat Rev Drug Discov. 2011;10(11):868-880

\title{
Thrombocytopathy and type 2B von Willebrand disease
}

\author{
Jerry Ware
}

Department of Physiology and Biophysics, University of Arkansas for Medical Sciences, Little Rock, Arkansas, USA.

\begin{abstract}
The knowledge gained from "experiments of nature" has always been paramount in identifying key players in pathophysiologic pathways. This is well characterized by naturally occurring bleeding and thrombotic disorders. In most cases, it is the absence of a particular protein that leads to recognition of its importance for normal physiology. On the other hand, gain-of-function mutations highlight not only the presence of the protein, but also how it regulates a particular physiologic response. In this issue of the JCI, Casari and colleagues define a previously unrecognized consequence of variant type $2 \mathrm{~B}$ von Willebrand factor (vWF) binding to blood platelets. More than 30 years after an initial description of type $2 \mathrm{~B}$ variant $\mathrm{vWF}$, the consequence of this spontaneous variant $\mathrm{vWF}$ binding to platelets is viewed as a dysregulation of platelet signaling pathways contributing to the type $2 \mathrm{~B}$ bleeding phenotype.
\end{abstract}

\section{Type 2B von Willebrand disease}

Among bleeding disorders, von Willebrand disease (vWD) is the most common. This inherited disorder is caused by production of a dysfunctional von Willebrand factor (vWF) (1). The current paradigm for the hemostasis regulation is that vWF circulates as a soluble multimeric protein with an indeterminate affinity for platelets or the intact vessel wall (2). Following vascular damage, surface-bound vWF is exposed in the subendothelium and captured from flowing blood to become a key bridging molecule, which supports platelet adhesion to an altered vascular surface. Thus, a simple explanation for the regulation of

Conflict of interest: The author has declared that no conflict of interest exists.

Citation for this article: J Clin Invest. 2013; 123(12):5004-5006. doi:10.1172/JCI73169.
vWF function is that soluble vWF has an unmeasurable affinity for platelets, while surface-bound vWF interacts efficiently with platelets. The importance of vWF and its platelet receptor, glycoprotein Ib-IX (GP-Ib-IX), is highlighted by well-characterized mutations that lead to the absence of vWF or GP-Ib-IX, resulting in bleeding phenotypes $(3,4)$. vWD constitutes a complex spectrum of clinical phenotypes that can be further subclassified based on levels of circulating protein, degree of protein multimerization, and functional interactions with platelets and coagulation factor VIII $(1,3)$.

One of the more intriguing vWD subtypes is the gain-of-function mutation designated vWD-type 2B (5). In this case, single amino acid mutations within the GP-Ib-IX-binding domain of vWF lead to an altered conformation that supports the soluble interaction of vWF with platelets in the circulation
(Figure $1 \mathrm{~A}$ and refs. 6, 7). Because the consequence of type $2 \mathrm{~B} \mathrm{vWF}$ is a spontaneous interaction with circulating platelets, the phenotypic outcome might be expected to be a prothrombotic. Instead, vWD-type $2 \mathrm{~B}$ leads to a counterintuitive phenotype - increased bleeding. The bleeding phenotype is thought to be promoted by the largest vWF multimers, which have the most hemostatic potential, being sequestered by platelets and unable to participate in hemostasis. The presumed consequences of soluble vWF binding to platelets are intravascular platelet clumping, platelet removal from the circulation, and varying degrees of thrombocytopenia. Thus, a combination of the absence of VWF with the most hemostatic potential and thrombocytopenia has historically been the explanation of the type $2 \mathrm{~B}$ bleeding phenotype.

While thrombocytopenia and the absence of high-molecular-weight vWF are reasonable explanations for type $2 \mathrm{~B}$ bleeding, the study by Casari et al. (8) in this issue of the JCI adds an additional explanation. Their current work suggests a third mechanism for type 2B bleeding; thrombocytopathy as a result of dysregulated platelet signaling is a consequence of variant $\mathrm{VWF}$ binding to platelets (Figure 1B). This dysregulated signaling impairs platelet aggregation, platelet secretion, and platelet spreading. For normal hemostasis, each of these platelet events is critical for the temporal sequence of events that support hemostasis. Specifically, Casari et al. provide evidence of 
A

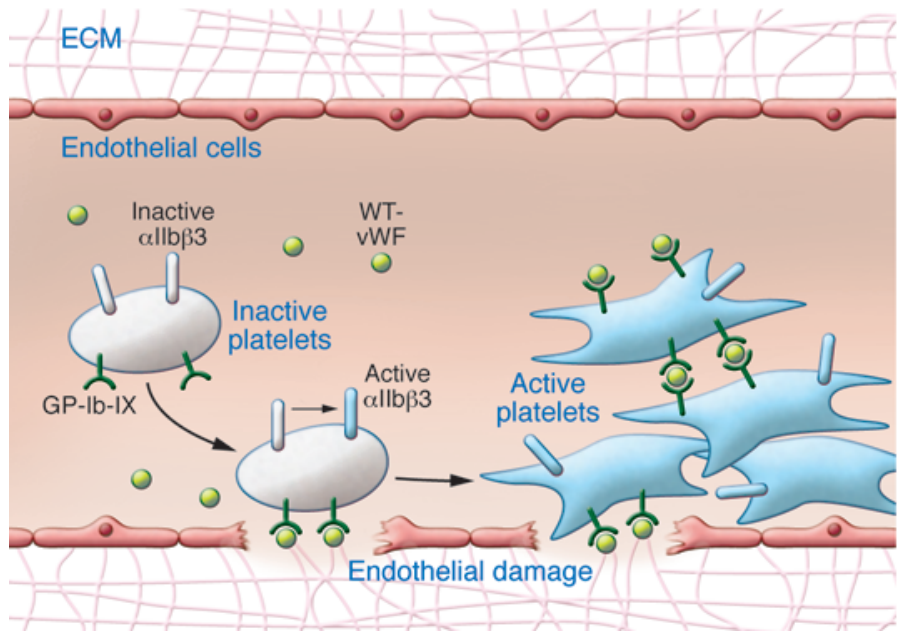

B

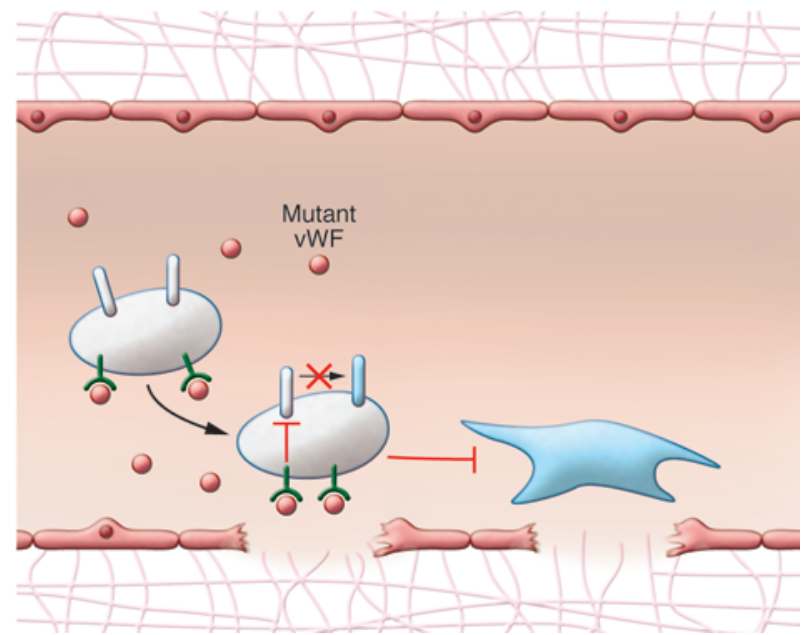

\section{Figure 1}

Regulation of the vWF/Platelet axis. (A) In response to endothelial cell damage, WT vWF binds to exposed extracellular matrix. This binding changes vWF affinity for the GP-Ib-IX, which promotes platelet adhesion. Platelet adhesion results in activation of integrins, including $\alpha \mathrm{llb} \beta 3$, which in turn promotes platelet spreading, and aggregation. (B) A mutant vWF associated with vWD-type 2B has increased affinity for platelet GP-Ib-IX. Mutant vWF prevents activation of $\alpha \mathrm{llb} \beta 3$, which reduces platelet adhesion, activation, and spreading in response to endothelial cell damage.

decreased activation of the platelet fibrinogen integrin receptor, $\alpha \operatorname{IIb} \beta 3$, as a consequence of type $2 \mathrm{~B} \mathrm{vWF}$ binding to platelet GP-Ib-IX (8). Indeed, decreased activation of $\alpha I I b \beta 3$ would significantly impact hemostasis. Thus, the work by Casari et al. provides an important mechanistic observation to explain bleeding associated with the type 2B phenotype more than 30 years after its recognition as a distinct subtype of vWD (5).

Thrombocytopathy associated with vWD-type 2B could also explain 2 additional features of the type $2 \mathrm{~B}$ phenotype. The first is a better explanation for why type $2 \mathrm{~B}$ does not result in some potential for microthrombi formation. Dysregulation of platelet signaling and reduced $\alpha \operatorname{IIb} \beta 3$ activation would support a type 2B-dependent antithrombotic mechanism. Second, a diagnositic feature of $v W D$-type $2 \mathrm{~B}$ is platelet agglutination at low doses of ristocetin, even though the overall aggregation may be reduced. Again, thrombocytopathy might be expected to reveal itself with an overall diminished platelet aggregation response, as observed using type $2 \mathrm{~B}$ plasma.

\section{Mutant vWF/GP-Ib-IX axis}

The quest to understand the molecular basis of the vWF/platelet GP-Ib-IX regulation has been a cornerstone of basic hemostasis research. Studies of vWD-type $2 \mathrm{~B}$ molecules have suggested that con- formational changes induced by mutations in vWF increase the platelet-binding affinity of vWF. For normal vWF, the surface-bound form is assumed to undergo key conformational changes that facilitate an increased affinity for platelet GP-Ib-IX. In the case of vWD-type $2 \mathrm{~B}$, the intrinsic mutation itself leads to an increased affinity for platelets (6). A similar increased affinity between vWF and platelet GP-IbIX can be caused by mutations in GP-IbIX that result in a phenotype described as "platelet-type vWD (Pt-vWD)" or "pseudo-vWD" $(9,10)$. Whether it is a gain-offunction mutation in the ligand (vWDtype $2 \mathrm{~B}$ ) or a mutation in the receptor (Pt-vWD), the net result is an increased bleeding risk. In support of the findings of Casari et al., earlier work on the molecular basis of Pt-vWD described a disruption of signaling pathways as a consequence of normal vWF binding to the mutant GP-IbIX receptor (11). Both cases pose the question of whether gain-of-function mutations in the ligand or receptor mimic the molecular events that occur during normal hemostasis or thrombosis. It is well documented that the vWF/GP-Ib-IX interaction between normal molecules precedes platelet activation and facilitates hemostasis. How the interaction of a mutant ligand or receptor in the vWF/GP-Ib-IX axis leads to signaling dysregulation awaits more detailed investigation.
Whether or not the interaction between vWF and platelets requires structural changes in both vWF and the platelet receptor, GP-Ib-IX, remains a long-standing debate. Exposure of vWF and platelets to a high-shear environment leads to a spontaneous interaction; however, it is less than clear whether this shear-induced interaction mimics those events occurring following vascular damage and surface-bound vWF. Solving the crystal structures of vWF in complex with the ligand-binding domain of GP-Ib-IX has used the type 2B mutations in vWF or the Pt-vWD mutation in GP-IbIX to facilitate complex cocrystallization (12). Considering the evidence that mutations in either the ligand or receptor are dysregulating platelet-dependent signaling events, we wonder whether these structures are telling us everything we should know about the molecular interactions in normal hemostasis. This is difficult to answer at this point and further confounded when it is recognized that all these structures have been determined using purified domains of vWF and GP-Ib-IX. The current structural analysis disregards any complementation that might occur from other domains of vWF or GP-Ib-IX.

\section{Antithrombotic approach}

The work by Casari and colleagues (8) may have implications for antithrombotic targeting of the vWF/GP-Ib-IX axis. As with 
any antithrombotic approach, the difficulty is finding efficacy without tipping the hemostatic balance to increased bleeding risk. Indeed, as a player in primary hemostasis, the vWF/platelet GP-Ib-IX axis has, to date, been a challenge to target without considerable risk. The findings by Casari et al. suggest an antithrombotic pathway mechanistic approach that has yet to be considered. While antagonizing the ligand/ receptor interaction could be justified for interrupting primary hemostasis, a type $2 \mathrm{~B}$ mimetic has potential to bind to normal platelet GP-Ib-IX and alter signaling pathways. Could such an approach lead to an antithrombotic effect beyond just blocking a ligand/receptor interaction? Could such a strategy lead to inhibition of activation pathways that mirror aspirin and/ or be complementary? While exploring this strategy is beyond the scope of the study by Casari et al., the identification of altered signaling as a consequence of mutant vWF binding to platelets suggests such an approach might be feasible. Casari and colleagues are to be commended for providing important data to explain molecular consequences for a gain-of-function mutation in vWF many years after the recognition of type $2 \mathrm{~B}$ as a distinct subtype of vWD.

\section{Acknowledgments}

I would like to thank Ginell Post and K.I. Varughese (University of Arkansas for Medical Sciences) for critical comments and helpful discussions.

Address correspondence to: Jerry Ware, University of Arkansas for Medical Sciences, 4301 W. Markham St., Little Rock, Arkansas 72205, USA. Phone: 501.526.6096; Fax: 501.686.8167; E-mail: jware@uams.edu.

1. Ruggeri ZM, Zimmerman TS. von Willebrand factor and von Willebrand disease. Blood. 1987;70(4):895-904.

2. Ruggeri ZM, Mendolicchio GL. Adhesion mechanisms in platelet function. Circ Res. 2007;100(12):1673-1685

3. James PD, Lillicrap D. The molecular characterization of von Willebrand disease: good in parts. $\mathrm{Br} \mathrm{J}$ Haematol. 2013;161(2):166-176.

4. Lopez JA, Andrews RK, Afshar-Kharghan V, Berndt MC. Bernard-Soulier syndrome. Blood.
1998;91(12):4397-4418

5. Ruggeri ZM, Pareti FI, Mannucci PM, Ciavarella N, Zimmerman TS. Heightened interaction between platelets and Factor VIII/von Willebrand factor in a new subtype of von Willebrand's disease. $N$ EnglJ Med. 1980;302(19):1047-1051.

6. Ware J, et al. Identification of a point mutation in type IIB von Willebrand disease illustrating the regulation of von Willebrand factor affinity for the platelet membrane glycoprotein Ib-IX receptor. Proc Natl Acad Sci U S A. 1991;88(7):2946-2950.

7. Celikel R, Ruggeri ZM, Varughese KI. von Willebrand factor conformation and adhesive function is modulated by an internalized water molecule. Nat Struct Biol. 2000;7(10):881-884.

8. Casari C, et al. von Willebrand factor mutation promotes thrombocytopathy by inhibiting integrin $\alpha I I b \beta 3$. J Clin Invest. 2013;123(12):5071-5081.

9. Murata M, Ware J, Ruggeri ZM. Site-directed mutagenesis of a soluble recombinant fragment of platelet glycoprotein Ib $\alpha$ demonstrating negatively charged residues involved in von Willebrand factor binding. J Biol Chem. 1991;266(23):15474-15480.

10. Othman M, Lopez JA, Ware J. Platelet-type von Willebrand disease update: the disease, the molecule and the animal model. Expert Rev Hematol. 2011;4(5):475-477.

11. Guerrero JA, et al. Visualizing the von Willebrand factor/glycoprotein Ib-IX axis with a platelettype von Willebrand disease mutation. Blood. 2009;114(27):5541-5546.

12. Huizinga EG, et al. Structures of glycoprotein Ib $\alpha$ and its complex with von Willebrand factor A1 domain. Science. 2002;297(5584):1176-1179.

\title{
BAFF-ling autoantibodies
}

\author{
Stefanie Sarantopoulos ${ }^{1}$ and Maureen A. Su²
}

1Department of Medicine, Division of Hematological Malignancies and Cellular Therapy, School of Medicine, Duke University, Durham, North Carolina, USA. ${ }^{2}$ Department of Pediatrics, Department of Microbiology and Immunology, School of Medicine, University of North Carolina at Chapel Hill, Chapel Hill, North Carolina, USA.

\begin{abstract}
There is emerging evidence that autoantibodies directed against cytokines modulate the severity of autoimmune disease. Identification of cytokine-targeted autoantibodies in patients can be informative for diagnosis and predicting clinical outcome. In this issue of the JCI, Price and colleagues used a multiplex protein microarray to identify autoantibodies in serum from SLE patients. They found autoantibodies directed against the $B$ cell-activating factor (BAFF) were associated with greater disease severity. This study highlights the contribution of cytokine-directed autoantibodies in disease and describes a valuable tool for identifying autoantibodies against serum antigens.
\end{abstract}

\section{Cytokine-targeting autoantibodies in disease}

Spontaneous autoantibody production is a hallmark of many autoimmune diseases, and these disease-specific autoantibodies are often useful in affirming a clinical autoimmune diagnosis. For instance, clinicians

Conflict of interest: The authors have declared that no conflict of interest exists.

Citation for this article: J Clin Invest. 2013; 123(12):5006-5008. doi:10.1172/JCI73166. test for autoantibodies against insulin and other pancreas-specific antigens to differentiate autoimmune (type 1) diabetes from other types of diabetes. In addition to serving as markers for autoimmunity, autoantibodies can play a central role in disease pathogenesis. Strong evidence exists that autoantibodies are important in development of SLE, a systemic autoimmune disease characterized by immune-mediated injury that affects a large number of tissues, including brain, blood vessels, and kidneys. Autoantibodies against nuclear antigens are important in disease initiation, directly mediating organ injury via complement-mediated cascades and other inflammatory mechanisms.

Interestingly, cytokines have now been described as autoantibody targets in a number of disease settings, resulting in a range of clinical manifestations (1). In many of these diseases, autoantibodies are produced against cytokines that are important in host defense and thus lead to functional immunodeficiency. Autoimmune polyendocrinopathy syndrome type 1 (APS-1), for example, is characterized by multiorgan autoimmunity and mucocutaneous candidiasis (2). For many years, the predisposition to candidiasis, which seemed to reflect a state of immunodeficiency, appeared to be an inconsistent finding among the multiple autoimmune manifestations in APS-1. A possible explanation for this seemingly irregular finding was 